\title{
Bewährtes und neue Trends im Überblick
}

\author{
J. Ruef \\ Medizinische Klinik III/Kardiologie, Universität Heidelberg \\ (Chefarzt: Prof. Dr. H.A. Katus)
}

Die Anwendung von Antikoagulanzien erfolgt interdisziplinär in einem breiten Indikationsspektrum. Dadurch gehören diese Präparate zu den am häufigsten verordneten Pharmaka. Dank ihres Einsatzes konnten in den vergangenen Jahren zahlreiche Thrombosen und Thromboembolien verhindert und die kardiovaskulär verursachte Morbidität reduziert werden. Trotz dieses Erfolges ist die Anwendung von Antithrombotika nicht unproblematisch und mit zum Teil gravierenden Nebenwirkungen behaftet. Daher wird die aktuelle Entwicklung von neuen antithrombotischen Substanzen mit Spannung verfolgt. Erwartet man von diesen doch im Vergleich zu den gängigen, bewährten Pharmaka eine höhere Effizienz, eine niedrigere Nebenwirkungsrate sowie eine einfachere Anwendbarkeit. Neue Trends in dieser Richtung (Pentasaccharid, Ximelagatran) könnten die Standards der Antikoagulation in absehbarer Zeit nachhaltig verändern. Dies betrifft folgende Indikationen: Prophylaxe und Therapie der venösen Thrombose und Lungenembolie, Schlaganfallprophylaxe bei Vorhofflimmern, Embolieprophylaxe bei Trägern künstlicher Herzklappen, Antikoagulation bei Schwangeren und die antithrombotische Therapie beim akuten Koronarsyndrom.

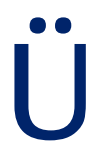

ber Jahrzehnte hinweg dominierten Vitamin-K-Antagonisten (VKA) und das unfraktionierte Heparin (UFH) das Feld der Antikoagulanzien, erst in den achtziger Jahren wurden die niedermolekularen Heparine (LMWH) eingeführt. Jetzt steht mit dem Pentasaccharid Fondaparinux ein neues Präparat zur Verfügung, das seine Effektivität bereits unter Beweis ge-

\section{Tab. 1 Nachteile der Vitamin-K-Antagonisten}

- nichtselektive Inhibition

- lange Halbwertszeit

- hohe Plasmaeiweißbindung

- Wechselwirkung mit Nahrung und Pharmaka

- Monitoring erforderlich

- Teratogenität

- Induktion von Coumarinnekrosen, Coumarinhepatitis stellt hat. Doch auch neue Substanzen - wie zum Beispiel der direkte Thrombininhibitor Melagatran und dessen oral applizierbares Prodrug Ximelagatran stehen kurz vor der Zulassung.

\section{Bewährte Antithrombotika Vitamin-K-Antagonisten}

Schon seit 65 Jahren ist der Wirkmechanismus der Vitamin-KAntagonisten bekannt: Die Coumarin-Derivate hemmen zwei Enzyme des Vitamin-K-Stoffwechsels und blockieren so die Bereitstellung von aktivem, reduzierten Vitamin K. Zur hepatischen Synthese der aktiven Gerinnungsfaktoren II, VII, IX und X sowie der Proteine $C$ und $S$ ist jedoch Vitamin K erforderlich.

Coumarin-Derivate können oral verabreicht werden, da sie aus dem Magen-Darm-Trakt ausgezeichnet resorbiert werden (das Phenprocou- mon beispielsweise hat eine Bioverfügbarkeit von mehr als 90\%). Die Plasmaeiweißbindung der Coumarin-Derivate ist sehr hoch, wodurch nur etwa $1 \%$ als freie aktive Substanz vorliegt. Dadurch erklärt sich einer der großen Nachteile dieser Substanzklasse (Tab. 1).

Denn schon geringe äußere Einflüsse - in erster Linie Komedikationen (z.B. Chemotherapeutika, Antiphlogistika oder Lipidsenker) - können die Konzentration des aktiven Wirkspiegels drastisch verändern. Wird nur 1\% der Substanz aus der Plasmaeiweißbindung verdrängt, verdoppelt sich die aktive Wirkstoffkonzentration. Andere Präparate können aber auch den Abbau der Vitamin-K-Antagonisten über das Zytochrom-P450-System hemmen oder aktivieren.

Mit dem Lebensalter ändert sich zudem der Bedarf an Coumarin, und es gibt genetische Variationen hinsichtlich des individuellen Bedarfs an VKA-Konzentrationen (43). Da auch das Blutungsrisiko im Alter immer höher wird, verschreiben Ärzte Vitamin-K-Antagonisten in solchen Fällen eher ungern - auch wenn die Indikation zur Antikoagulation besteht (15).

Die wichtigsten Nebenwirkungen dieser Therapeutika sind Blutungskomplikationen, betroffen sind etwa $3-5 \%$ der Patienten. Besonders gefürchtet sind intrazerebrale Blutungen mit einer Prävalenz von 0,2-0,5 Ereignissen pro $100 \mathrm{~Pa}-$ tientenjahren und einer Mortalität 
von 70-80\% (32). Bei der Einleitung der Therapie können sich Coumarinnekrosen oder rezidivierende Hepatitiden entwickeln, zudem existiert ein teratogener Effekt im ersten Schwangerschaftstrimenon.

\section{Heparin}

Bis in die 80er Jahre galt unfraktioniertes Heparin (UFH) als Goldstandard in der temporären Thromboseprophylaxe und -therapie. Mittels einer niedrig dosierten („lowdose“) Heparinbehandlung (5 000 IE unfraktioniertes Heparin zwei- bis dreimal täglich subkutan) konnte die perioperative Thrombosehäufigkeit gegenüber unbehandelten Kontrollgruppen um bis zu 60\% gesenkt werden (7). Bei manifester Thrombose wurde unfraktioniertes Heparin klassischerweise als Dauerinfusion verabreicht und der antikoagulatorische Effekt anhand der aPTT gemessen.

Inzwischen hat das fraktionierte, niedermolekulare („low molecular weight heparin“; LMWH) das unfraktionierte Heparin weitgehend ersetzt. Dies betrifft insbesondere die orthopädische und chirurgische, aber auch die internistische Thromboseprophylaxe, da die neueren Substanzen die relativen Thromboseraten gegenüber unbehandelten Kontrollgruppen um bis zu 70\% reduzieren können (33-35).

Die negativ geladenen Sulfatgruppen - vor allem der langen Heparinketten des unfraktionierten Heparins - bewirken eine starke Bindung an Plasmaeiweiße und Zelloberflächen. Einige dieser Proteine steigen bei Akutphasereaktionen an oder werden bei thrombotischen Prozessen ausgeschüttet (Plättchenfaktor 4, von-Willebrand-Faktor). Dies ist - unter anderem - die Ursache, warum sich die antikoagulatorische Intensität von unfraktioniertem Heparin individuell unterscheidet, sodass ein regelmäßiges Monitoring unerlässlich ist.

Heparin ist der klassische Vertreter eines indirekten und unselektiven Faktor-Xa-Inhibitors. Es entfaltet seine antikoagulatorische Wirkung (vornehmlich gegenüber Faktor Xa und IIa) indirekt über Antithrombin, an welches das Heparin bindet. Die Hemmung von Thrombin
(IIa) erfordert die Bindung von mindestens 18 Saccharidresten des Heparins an Antithrombin, für die Hemmung des Faktors Xa dagegen reichen fünf Saccharidreste (5). Daher vermitteln niedermolekulare Heparine aufgrund der vorwiegend kurzkettigen Moleküle vermehrt eine anti-Xa-Wirkung, während unfraktioniertes Heparin Xa und IIa gleichwertig hemmt.

Niedermolekulare Heparine verursachen im Vergleich zu unfraktioniertem Heparin seltener eine heparininduzierte Thrombozytopenie (HIT), wirken weniger aktivierend auf Thrombozyten, aktivieren aber den so genannten "tissue factor pathway inhibitor" (TFPI) und sind deutlich weniger bis überhaupt nicht plazentagängig. Ein wesentlicher Vorteil der niedermolekularen Heparine ist zudem die subkutane Applikationsmöglichkeit - auch bei therapeutischer Anwendung. Dies ermöglicht eine patientenfreundliche ambulante Therapie und reduziert die Kosten (19).

Trotzdem haben auch die niedermolekularen Heparine noch gewisse Nachteile: Sie lassen sich nur parenteral applizieren, die Anwendung muss bei therapeutischer Dosierung zweimal täglich gewichtsadaptiert erfolgen und aufgrund des heterogenen Molekülgemisches inklusive Trägerlösungen sind allergische Reaktionen nicht selten (Tab. 2). Darüber hinaus vermag Heparin aufgrund seines indirekten Wirkmechanismus via Antithrombin nicht, prothrombokinasegebundenen Faktor Xa und fibringebundenes Thrombin zu inhibieren.

\section{- Neue Antithrombotika}

Das Gerinnungssystem mit seiner Aktivierungskaskade bietet verschiedene Ziele für spezifische und selektive Pharmaka (Abb. 1). An zentraler Stelle zwischen der intrinsischen und extrinsischen Gerinnungskaskade steht der Faktor Xa. Hier ist zudem ein Verstärkermechanismus in der Koagulationskaskade eingebaut. Pro Minute kann ein Molekül Xa innerhalb des Prothrombinasekomplexes 138 Moleküle Thrombin generieren. Daher ist der Faktor Xa eines der Haupt-

\section{Tab. 2 Nachteile der Heparine}

- Aufreinigung aus biologischer Masse

- nichtselektive Inhibition

- heparininduziertre Thrombozytopenie, Interaktion mit Plättchenfaktor 4

- Aktivierung der Thrombozytenaggregation

- ausschließlich renale Elimination (LMWH)

- Induktion von Allergien

- gewichtsabhängige Dosierung (vor allem bei therapeutischer Anwendung)

ziele der pharmakologischen Interventionen.

Als Bindeglied zwischen der plasmatischen und zellulären Gerinnung ist das Thrombin ein weiteres wichtiges Zielmolekül - es repräsentiert den potentesten physiologischen Aktivator der Thrombozytenaggregation. Im Thrombus gebundenes Thrombin ist enzymatisch aktiv, daher ist die Blockade dieses fibringebundenen Thrombins mittels kleinmolekularer, möglichst reversibler Pharmaka, ein vorrangiges Ziel.

Die Gerinnungskaskade lässt sich über den extrinsischen Weg mittels des „tissue factor pathway inhibitor“ (TFPI), Antikörpern gegen den Gewebefaktor und Inhibitoren des VIIa/TFKomplexes hemmen (Abb. 1) (39). Zu den Letzteren gehört unter anderem auch rNAPc2 („recombinant nematode anticoagulant protein“). Dieses aus dem Hakenwurm Ancylostoma caninum gewonnene Antikoagulans war sowohl in einer orthopädischen Thromboseprophylaxestudie (26) als auch in einer Studie zur elektiven Koronarangioplastie (29) wirksam. Dank seiner langen Halbwertszeit reicht eine Injektion von rNAPc2 jeden zweiten Tag aus.

\section{Faktor-Xa-Inhibitoren}

Das zur Konfromationsänderung von Antithrombin notwendige Pentasaccharid konnte inzwischen in einer aufwändigen Synthesekette hergestellt werden. Da diese Substanz anders als die Heparine selektiv für Faktor Xa ist, ist sie ein Vertreter einer neuen Klasse indirekter Faktor-Xa-Inhibitoren. Mithilfe von Modifikationen an den Seitenketten der Zuckermoleküle können Pentasaccharide mit unterschiedlichen Eigenschaften hergestellt werden. 
Derzeit sind zwei Substanzen näher charakterisiert: Fondaparinux (Arixtra ${ }^{\circledR}$ ) und Idraparinux. Wie Heparin müssen die Pentasaccharide parenteral appliziert werden. In einer Serie von randomisierten Studien zeigte sich Fondaparinux gegenüber niedermolekularen Heparinen bei der Thromboseprophylaxe von Patienten nach orthopädischen Operationen überlegen (4, 45). Obwohl Fondaparinux anders als niedermolekulares Heparin erst postoperativ zum Einsatz kam, konnte das relative Thromboserisiko in diesem Hochrisikokollektiv um $50 \%$ reduziert werden. Allerdings beziehen sich diese Resultate auf asymptomatische Venenthrombosen.

Seit 2002 ist Fondaparinux zur Thromboseprophylaxe bei orthopädischen Operationen zugelassen. Täglich werden hierzu einmal 2,5 mg der Substanz subkutan appliziert, die Plasmahalbwertszeit von Fondaparinux beträgt etwa 18 Stunden. Da das Pentasaccharid - ebenso wie niedermolekulares Heparin fast ausschließlich renal eliminiert wird, sollte bei eingeschränkter Nierenfunktion (Clearance unter 40 $\mathrm{ml} / \mathrm{min}$ ) die Dosis angepasst oder auf andere Präparate (z.B. unfraktioniertes Heparin) ausgewichen werden.

\section{Abb. 1 Antithrombotika und ihre Angriffsorte im Gerinnungssystem}

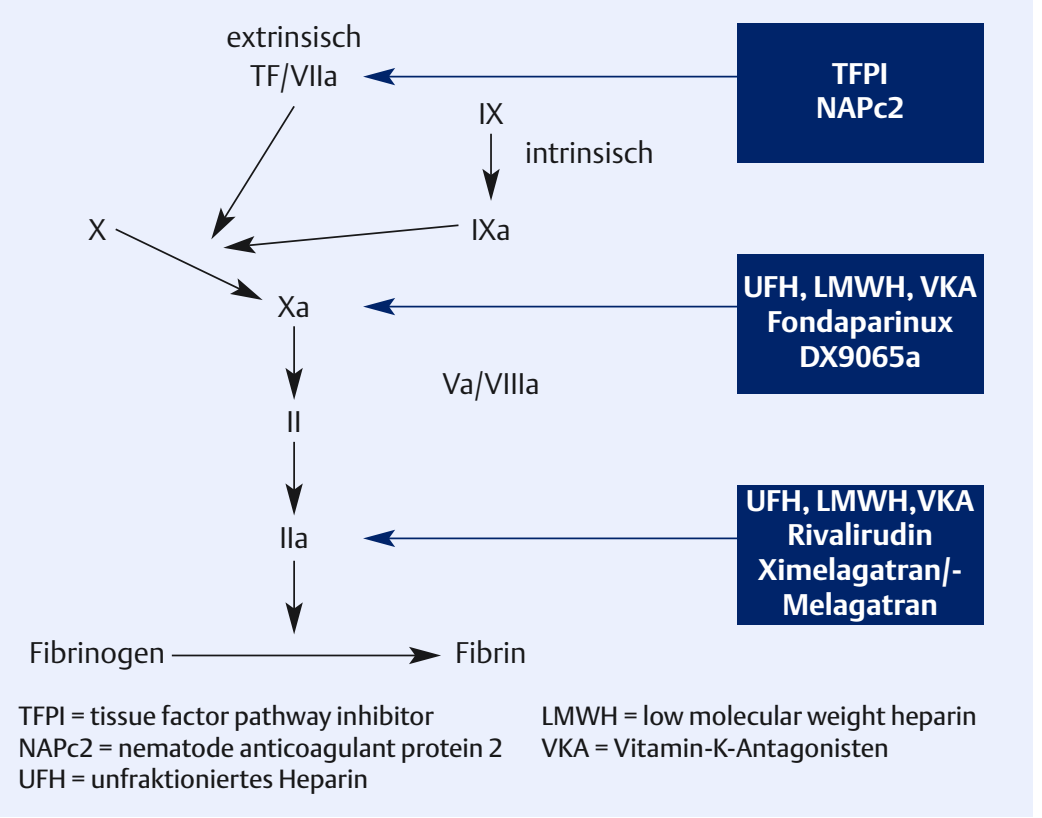

Inzwischen ist Fondaparinux zur verlängerten postoperativen orthopädischen Thromboseprophylaxe zugelassen (PENTHIFRA-PLUS, 12). Auch in der Abdominalchirurgie wurde Fondaparinux getestet und erwies sich mit einer postoperativen Dosierung einer perioperativen Thromboseprophylaxe mit Dalteparin als gleichwertig (PEGASUS1Studie, 2).

Für die internistische Thrombolekularen Heparinen derzeit lediglich Enoxaparin zugelassen, Grundlage sind die Ergebnisse der MEDENOX $^{2}$-Studie (4). In einer vergleichbaren Erhebung konnte jetzt auch zebo die thromboembolischen Ereignisse um 50\% reduzieren. Mit $0,2 \%$ waren die Blutungskomplikationen eher selten (ARTEMIS ${ }^{3}$-Studie). Aufgrund dieser Daten soll nun die Zulassung zur internistischen Thromboseprophylaxe beantragt werden.

Erste Studienergebnisse mit Fondaparinux liegen zudem zur Thrombosetherapie vor. In den MATISSE4-Studien zur symptomatischen tiefen Venenthrombose und Lungenembolie wurde Fondaparinux (einmal 7,5 mg s.c/die) mit Enoxaparin (zweimal täglich gewichtsseprophylaxe ist von den niedermodas Fondaparinux gegenüber Pla- adaptiert) bzw. mit unfraktioniertem Heparin verglichen. Die Substanzen waren hinsichtlich Effektivität und Sicherheit vergleichbar (44). Daher wird die Zulassung von Fondaparinux zur Thrombosetherapie in den USA bis Ende 2003 erwartet. Mit $\mathrm{OASIS}^{5}$ gibt es zudem ein großes multizentrisches Studienprogramm, das den Stellenwert von Fondaparinux beim akuten Koronarsyndrom untersucht, nachdem erste Studien (Phase IIa) bei instabiler Angina pectoris (PENTUA6-Studie) und bei Thrombolyse des akuten Myokardinfarktes (PENTALYSE7-Studie) viel versprechende Ergebnisse lieferten (40).

Das Pentasaccharid Idraparinux hat aufgrund einer zusätzlichen Bindungsstelle zu Antithrombin eine lange Halbwertszeit von etwa 80 Stunden. Daher eignet sich die Substanz für eine längerfristige Anwendung mit einer Injektion einmal pro Woche. In einer Phase-IIa-Studie $\left(\right.$ PERSIST $\left.^{8}\right)$ reduzierte Idraparinux in einer Dosierung von $2,5 \mathrm{mg} /$ Woche nicht nur Thromboembolien effizienter als Warfarin ( 0,6 versus $1,6 \%$ ). Auch bezüglich der Mortalität schnitt Idraparinux besser ab - und dies ohne vermehrte Blutungskomplikationen (36). Derzeit wird Idraparinux in der längerfristigen Sekundärprophylaxe nach tiefer Venenthrombose oder Lungenembolie gegen INR-adjustierte Vitamin-K-Antagonisten (VAN-GOGH-Studienprogramm) sowie in der Thromboembolieprophylaxe bei Vorhofflimmern (AMADEUS-Studie) getestet.

Derzeit befinden sich auch eine Reihe von direkten Faktor-Xa-Inhibi-

\footnotetext{
${ }^{1}$ pentasaccharide in general surgery study

2 medical patients with enoxaparin

3 arixtra for thromboembolism prevention in a medical indications study

4 fondaparinux (Arixtra) in comparison to (low molecular weight) heparin for the initial treatment of symptomatic deep venous thrombosis or pulmonary embolism

${ }^{5}$ organization to assess strategic for ischemic syndromes

${ }^{6}$ pentasaccharide in unstable angina

${ }^{7}$ pentasaccharide as an adjunct to fibrinolysis in ST-elevation acute myocardial infarction

8 a novel long-acting synthetic factor $X a$ inhibitor (Idraparinux Sodium) to replace warfarin for secondary prevention in deep vein thrombosis
} 
toren in der Entwicklung. Am weitesten vorangeschritten sind die präklinischen und klinischen Tests für DX-9065a. Im Tiermodell erwies sich die Substanz als effektiv bezüglich der Prävention von venösen und arteriellen Thrombosen (17). Erste klinische Erfahrungen wurden bei Patienten mit stabiler koronarer Herzerkrankung erhoben (11). Aufgrund der guten enteralen Resorption kann DX-9065a nicht nur parenteral, sondern auch oral appliziert werden.

\section{- Direkte Thrombininhibitoren}

Klassische Vertreter direkter Thrombininhibitoren sind die Hirudine. Studiendaten zufolge sind Hirudine bei der Prophylaxe von Thrombosen ebenso effektiv wie Heparine. Allerdings weisen sie auch eine höhere Nebenwirkungsrate auf. Daher beschränkt sich die Zulassung der Hirudinderivate Lepirudin (Refludan ${ }^{\circledR}$ ) und Bivalirudin (AngioMax $^{\circledR}$ ) sowie des kleinmolekularen Thrombininhibitors Argatroban (Avoca ${ }^{\circledR}$ ) auf die Thromboseprophylaxe und -therapie bei heparininduzierter Thrombozytopenie sowie auf die arterielle Thromboseprophylaxe bei der Koronarangioplastie (Bivalirudin und Argatroban sind derzeit lediglich in den USA zugelassen).

Das semisynthetische Hirudinfragment Bivalirudin konnte kürzlich in einer Myokardinfarktstudie eine dem Heparin gleichwertige antithrombotische Potenz belegen (27). Dieses Konzept der arteriellen antithrombotischen Therapie bei Myokardinfarkt mit Substitution von Heparin durch Bivalirudin überprüft derzeit die ACUITY9-Studie.

\section{Melagatran/Ximelagatran}

Eine viel versprechende Alternative ist der reversible, direkte Thrombininhibitor Melagatran und dessen oral applizierbares Prodrug,

${ }^{9}$ acute catherization and urgent intervention thrombotic strategy

10 melagatran for thrombin inhibition in orthopaedic surgery

11 expanded prophylaxis evaluation surgery study

12 oral direct thrombin inhibitor ximelagatran for venous thromboembolism

13 stroke prevention by oral thrombin inhibitor in atrial fibrillation das Ximelagatran. Dieses Dipeptid blockiert reversibel das katalytische Zentrum von Thrombin. Während Melagatran als wenig lipophiles und geladenes Molekül kaum enteral resorbiert wird, weist das Prodrug Ximelagatran mit einer 170-fach höheren Lipophilität eine orale Bioverfügbarkeit von $20 \%$ auf, wodurch eine stabile, köpergewichtsunabhängige Plasmakonzentration gewährleistet wird (20). Bei einer Plasmahalbwertszeit von ungefähr drei bis vier Stunden muss Ximelagatran zweimal täglich oral verabreicht werden. Aufgrund des stabilen Wirkspiegels entfällt ein Monitoring der antikoagulatorischen Wirkung. Im Falle einer dennoch gewünschten Therapiekontrolle kann die Ecarinzeit bestimmt werden - ein erster kommerzieller chromogener Assay hierfür steht bereits zur Verfügung (ECA-T, Haemochrom Diagnostica).

Metaboliten von Melagatran sind nicht bekannt. Die Substanz wird weitgehend renal ausgeschieden, weshalb sie bei reduzierter Nierenfunktion wahrscheinlich nur mit Einschränkungen appliziert werden kann. Interaktionen von Ximelagatran/Melagatran mit der Nahrung oder mit Begleitmedikamenten können bisher ausgeschlossen werden. Laut dem METHRO10_ und EXPRESS ${ }^{11}$-Studienprogramm ist eine Kombination aus perioperativ subkutan verabreichtem Melagatran (vor der Operation $2 \mathrm{mg}$, nach der Operation $3 \mathrm{mg}$ s.c.), gefolgt von oralem Ximelagatran (zweimal $24 \mathrm{mg}$ / die) einer Thromboseprophylaxe mit Enoxaparin überlegen und gleichzeitig bezüglich Blutungen weitgehend sicher (13). So konnte das relative Risiko proximaler tiefer Venenthrombosen und Lungenembolien um über $60 \%$ reduziert werden $(6$, 18).

Auch die Gabe von Ximelagatran zur Sekundärprophylaxe nach tiefer Venenthrombose (TVT) wurde bereits getestet: In der THRIVE ${ }^{12}$ III-Studie wurde nach einer tiefen Venenthrombose regulär über sechs Monate mit Vitamin-K-Antagonisten antikoaguliert. Anschließend erhielten die Patienten über $18 \mathrm{Mo-}$ nate entweder Ximelagatran (zweimal 24 mg oral/die) oder Plazebo. Im
Verumarm reduzierte sich das relative Risiko tiefer Venenthrombosen um $84 \%$, die Blutungskomplikationen stiegen dabei nicht signifikant (14).

Bei akuter Thrombose konnte Ximelagatran (2 x 36 mg) im Vergleich zur Standardtherapie mit Enoxaparin gefolgt von Vitamin-KAntagonisten (THRIVE-treatmentStudie) die Zahl an Rethrombosen, die Mortalität sowie die Blutungskomplikationen leicht senken. Der Unterschied erreichte allerdings keine statistische Signifikanz (25). Damit ist die Substanz der Standardtherapie ebenbürtig. Da das Medikament im Vergleich zur herkömmlichen oralen Antikoagulation aber eine wesentlich einfachere Anwendung verspricht, bedeutet dies bereits einen Fortschritt.

Einen weiteren Erfolg konnte Ximelagatran in einem anderen wichtigen Indikationsgebiet für längerfristige Antikoagulation erzielen, dem Vorhofflimmern. In der unverblindeten SPORTIF13-III-Studie bei Patienten mit nichtvalvulärem Vorhofflimmern erzielte Ximelagatran (zweimal $36 \mathrm{mg} / \mathrm{die}$ ) in der Ontreatment-Analyse hinsichtlich der Studienendpunkte Apoplex und/ oder systemische Embolisation gegenüber der Therapie mit VitaminK-Antagonisten eine relative Risikoreduktion von $41 \%$. Nimmt man noch den dritten Studienendpunkt „Tod“ hinzu, bleibt immerhin noch eine Risikoreduktion von $25 \%$ bestehen. Von den Patienten unter VKA waren $81 \%$ im therapeutischen $\mathrm{Be}$ reich einer INR 2,0-3,0, was als ungewöhnlich gut einzuschätzen ist (21).

Die Zulassung von Ximelagatran/Melagatran zur Prophylaxe von Venenthrombosen unter dem

\section{Tab. 3 Vorteile von Pentasaccharid}

- selektiver Inhibitor

- synthetische Herstellung mit homogenen Chargen

- geringe Wechselwirkung mit Nahrung und Pharmaka

- keine Induktion einer Thrombozytopenie

- keine Thrombozytenaktivierung

- Anwendung nur einmal täglich

- keine Metabolisierung 
Handelsnamen Exanta ${ }^{\circledR}$ wurde im Sommer 2002 in Europa beantragt. Aufgrund der neuen Studiendaten von SPORTIF III wird für Ende 2003 zusätzlich ein Antrag auf Zulassung zur Schlaganfallsprophylaxe bei Vorhofflimmern vorbereitet, die Indikation „Behandlung akuter Venenthrombosen" wird sicher folgen. Definierte Dosierrichtlinien liegen noch nicht vor (voraussichtlich zweimal $24 \mathrm{mg} /$ die oral zur Prophylaxe der tiefen Venenthrombose, zweimal $36 \mathrm{mg} /$ die zur Thrombosetherapie und Schlaganfallprophylaxe).

Die Vision einer oralen Antikoagulation mit breiterem therapeutischem Fenster als bei den VitaminK-Antagonisten und dem potenziellen Wegfall der Notwendigkeit eines Therapiemonitorings mit dadurch erheblicher Kostenreduktion erscheint viel versprechend. Ximelagatran scheint so insgesamt die Bedingungen für ein ideales Antithrombotikum zu erfüllen (Tab. 4).

Der derzeit einzige erkennbare Nachteil von Melagatran/Ximelagatran ist ein Anstieg des Leberenzyms GPT (ALAT) auf über das dreifache der Norm, wovon 10-15\% der Patienten betroffen sind. Dieser Anstieg ist jedoch auch unter Fortführung der Therapie reversibel. Bisher konnten weder Symptome noch Nachteile irgendeiner Art mit diesem Enzymanstieg in Zusammenhang gebracht werden. Auch die genaue Ursache bleibt bislang unklar. Daher sollten bei einer zukünftigen Anwendung von Ximelagatran in den ersten Wochen der Behandlung Leberenzymkontrollen erfolgen

\section{Tab. 4 Das ideale Antithrombotikum}

- selektive Inhibition

- parenterale und orale Applikation

- fixe Dosierung

- schneller on- und offset

- stabile Pharmakokinetik mit geringer Plasmaeiweißbindung

- keine Interaktion mit Nahrungsmitteln oder Medikamenten

- kein Monitoring nötig

- hohe therapeutische Breite

- keine Metabolisierung

- Inhibition von freiem und fibringebundenem Thrombin
- auch wenn deren Anstieg offensichtlich keine pathophysiologische Relevanz hat oder den Abbruch der Therapie notwendig macht.

\section{Alternativen bei aus- gesuchten Indikationen}

Problematisch ist eine Antikoagulation bei Patienten mit mechanischen Herzklappenprothesen. Bislang ist die Datenlage bezüglich Effektivität und Sicherheit für eine Heparintherapie noch nicht vollständig geklärt. Erst vor kurzem wurde gezeigt, dass das Enoxaparin eine orale Antikoagulation bei Patienten mit mechanischen Klappenprothesen - sicher und effektiv ersetzen kann. Dieses Ergebnis ist umso erstaunlicher, da $74 \%$ der Patienten einer Hochrisikogruppe angehörten (Mitralprothese oder Aortenprothese mit zusätzlichen Risikofaktoren, 16).

Inzwischen wurden bei einem Patient mit mechanischer Herzklappenprothese erste Daten zur längerfristigen Thromboembolieprophylaxe mit Fondaparinux erhoben. Thromboembolien oder Blutungskomplikationen wurden bei der Behandlung nicht beobachtet (22). Ob sich bei diesen Indikationen auch Ximelagatran/Melagatran eignet, bleibt abzuwarten.
Zum Teil problematisch ist auch die Anwendung von Antikoagulanzien während der Schwangerschaft: So sind Vitamin-K-Antagonisten im ersten Trimenon kontraindiziert, auch von der Gabe von Heparinoiden oder Hirudinen wird aufgrund mangelnder Daten abgeraten. Bewährt hat sich die Gabe von unfraktioniertem oder niedermolekularem Heparin. Erste Daten bezüglich Ximelagatran/Melagatran belegen, dass die Substanz - ähnlich wie Vitamin-KAntagonisten - nicht in signifikanten Mengen in die Muttermilch übertritt (24). Ausführliche Untersuchungen zu den Effekten von Pentasaccharid oder Ximelagatran bzw. Melagatran während der Schwangerschaft stehen allerdings noch aus.

\section{Neue Konzepte}

Für die Standardantikoagulation nach tiefer Beinvenenthrombose (TVT) oder Lungenembolie wird je nach Lokalisation, Häufigkeit oder Thrombophiliestatus eine Dauer zwischen sechs Wochen bis dauerhaft gewählt und eine INR 2,0-3,0 angestrebt. Unter diesen Bedingungen konnten die Vitamin-K-Antagonisten bisher keine befriedigende Risiko-Nutzen-Ergebnisse bei der Langzeitanwendung erzielen. Das

\section{Kasuistik}

K onsiliarisch wird die Thrombophilieambulanz von der Hepatitissprechstunde konsultiert bezüglich eines 39jährigen Patienten mit klinischen und laborchemischen Zeichen einer Hepatitis. Etwa acht Monate zuvor war nach einer spontanen tiefen Beinvenenthrombose eine orale Antikoagulation mit Phenprocoumon (Marcumar ${ }^{\circledR}$ ) begonnen worden. Im Thrombophiliescreening hatte sich eine homozygote Faktor-V-Leidenmutation ergeben, woraufhin die Indikation zur lebenslangen Antikoagulation gestellt wurde. Bei fehlenden anderen Ursachen der Hepatitis (Virushepatitis, Begleitmedikation etc.) wurde Phenprocoumon abgesetzt.

Nach Umstellung auf nierdermolekulares Heparin traten nach Verwendung von drei verschiedenen Präparaten allergische Hautreaktionen auf. Es wurde daraufhin nach Normalisierung der Transaminasen eine erneute orale Antikoagulation mit Acenocoumarol (Sintrom ${ }^{\circledR}$ ) eingeleitet. Nach acht Wochen stiegen die Transaminasen im Sinne einer Hepatitis erneut an. Bei offensichtlicher hepatotoxischer Kreuzreaktivität der Vitamin-K-Antagonisten wurde die orale Antikoagulation beendet und die Antikoagulation mit Pentasaccharid (Arixtra ${ }^{\circledR}$ ) begonnen.

Zwar ist die coumarininduzierte Hepatitis äußerst selten, doch diese Fallstudie macht deutlich, dass die bisher verfügbaren Standardantikoagulanzien Limitationen aufweisen. So ist es wünschenswert, dass bei einer gleichzeitigen Unverträglichkeit von Heparinen und Vitamin-K-Antagonisten neue, gut verträgliche Antikoagulanzien zur Verfügung stehen. 
Risiko für eine rekurrente Thrombose wird mit jährlich 6-9\% angegeben - unabhängig davon, wie lange zuvor therapiert wurde (3). Dies hat zu Überlegungen geführt, ob nicht eine dauerhafte Antikoagulation mit Vitamin-K-Antagonisten sinnvoll ist.

Aufgrund des bei der standardisierten Durchführung (INR 2,0-3,0) nicht unerheblichen Blutungsrisikos ging die PREVENT14-Studie der Frage nach, ob eine niedrig dosierte Antikoagulation in der langfristigen Sekundärprophylaxe der tiefen Venenthrombose erfolgreich ist. Patienten wurden im Anschluss an eine Standardantikoagulation mit niedrig dosiertem Warfarin therapiert (INR 1,5-2,0), verglichen wurde gegen Plazebo. Geplant war eine Behandlung über vier Jahre, die Studie wurde jedoch aufgrund der positiven Ergebnisse in der Warfaringruppe nach einer durchschnittlichen Behandlungszeit von zwei Jahren vorzeitig abgebrochen (Risikoreduktion 64\%, 37).

Diese Ergebnisse haben die Diskussion um Vor- und Nachteile einer langfristigen Antikoagulation erneut entfacht. Jedoch sollte nicht unerwähnt bleiben, dass die Hauptzahl der Thrombosen in der Plazebogruppe in den ersten zwei Monaten nach Absetzen der Standardantikoagulation auftraten. Dies deckt sich mit zahlreichen anderen Beobachtungen und ist möglicherweise ein für Vitamin-K-Antagonisten spezifisches Problem der Reboundhyperkoagulabilität (31). Hier könnten neue Antithrombotika (z.B. Idraparinux oder Ximelagatran) eine wirksame Alternative sein.

In den letzten Jahren kam auch erneut die Frage auf, ob eine Antikoagulation nach Myokardinfarkt effektiv und sicher ist. Schon in den 80er Jahren verglichen kleine Studien Acetylsalicylsäure und die orale Antikoagulation in dieser Indikation, ohne dass sich ein signifikanter Unterschied ergeben hätte. Und

\footnotetext{
14 long-term, low-intensity warfarin therapy for the prevention of recurrent venous thromboembolism

15 anticoagulants in the secondary prevention of events in coronary thrombosis

16 warfarin-aspirin re-infarction study
}

auch eine Kombination einer niedrig dosierten Antikoagulation (INR > 1,5) mit Aspirin ist nicht besser als Aspirin alleine, wie Daten aus den 90er Jahren zeigen (28).

In der ASPECT ${ }^{15}$-II-Studie dagegen war sowohl eine alleinige Antikoagulation (INR 2,0-3,0) als auch eine Kombination der Antikoagulation mit ASS einer alleinigen Behandlung mit ASS überlegen (46). Prinzipiell hat die WARIS16-II-Studie diese Ergebnisse bestätigt (10). Allerdings zeigten beide Studien eine Zunahme der Blutungskomplikationen unter der Kombinationstherapie. Ob die genannten neuen Antithrombotika günstigere Kombinationspartner oder sogar Monotherapeutika bei dieser Indikation sein können, bleibt derzeit abzuwarten.
Ein weiteres wichtiges Indikationsgebiet der Antikoagulation ist die Schlaganfallprophylaxe bei Vorhofflimmern, die ein sechsfach erhöhtes Schlaganfallrisiko darstellt. Risikopatienten profitieren von der Antikoagulation, während bei niedrigem Risiko und niedrigem Lebensalter auch Acetylsalicylsäure indiziert sein kann (23). Aber gerade die älteren Risikopatienten haben unter der oralen Antikoagulation mit Vitamin-K-Antagonisten ein erhöhtes Blutungsrisiko.

Da bei Vorhofflimmern eine Rhythmisierung nicht besser ist als das Verbleiben im Vorhofflimmern mit Frequenzkontrolle (42) und die in den Sinusrhythmus konvertierten Patienten wegen eines möglichen okkulten intermittierenden Vor-

\section{Glossar}

\section{Faktor Xa}

zentraler Faktor der Gerinnungskaskade: Die Gerinnungskaskade wird in der Regel durch Gewebefaktor („tissue factor“, TF) aktiviert, einem transmembranösen Rezeptor, der aktivierten Faktor VII (VIla) bindet. Dieser Komplex aktiviert Faktor IX und X. Faktor Xa wiederum formt mit Va und VIIla den Prothrombinasekomplex an Membranoberflächen (Endothelzellen, Thrombozyten). Dieser Komplex schließlich konvertiert Prothrombin (II) in Thrombin (Ila), letzteres wandelt Fibrinogen in Fibrin um (Abb. 1)

\section{Heparine}

klassische Vertreter eines indirekten und unselektiven Faktor-Xa-Inhibitors. Unfraktioniertes Heparin (UFH) ist ein heterogenes Gemisch von Polysaccharidketten aus 18-100 Monosacchariden (5 000-30 000 Dalton), die niedermolekularen Heparine (LMWH = „low molecular weight heparin“) bestehen aus Kettenfragmenten (10-30 Monosaccharideinheiten, 3000-9 000 Dalton)

\section{Hirudin}

ein aus 65 Aminosäuren zusammengesetztes, aus dem Blutegel isoliertes Antikoagulans. Hirudine sind klassische selektive Thrmobininhibitoren, die am aktiven Zentrum und der Fibrinogenbindungsstelle des Thrombinmoleküls bivalent binden und so Thrombin irreversibel inhibieren

\section{Hyperkoagulabilität}

gesteigerte Gerinnbarkeit des Blutes, die graduell unterschieden wird. Sie entsteht entweder durch eine Vermehrung gerinnungsfördernder Faktoren (Prokoagulanzien), durch eine Verminderung gerinnungshemmender Faktoren oder durch die Präsenz aktivierter Gerinnungsprofaktoren in der Blutbahn

\section{Pentasaccharide}

synthetisch hergestellte selektive Faktor-Xa-Inhibitoren (Fondaparinux bzw. Idraparinux) aus fünf Monosaccharidresten

\section{Tissue Factor}

der physiologische Initiator der Blutgerinnung. Die Lokalisierung des Tissue Factors in der Gefäßwand verhindert in einem intakten Gefäß den Gerinnungsstart 
hofflimmerns von einer Antikoagulation profitieren dürften, wird sich in Zukunft die Zahl der eine Antikoagulation benötigenden Patienten wohl erhöhen. Unter diesem Gesichtpunkt wirken die guten Ergebnisse von Ximelagatran aus der SPORTIF-Studie ermutigend, da ein viel versprechendes alternatives Therapiekonzept in Sichtweite rückt.

\section{Anticoagulation - Current Standards and New Trends \\ Anticoagulants are applied in several} medical specialties for a variety of indications. Therefore anticoagulants are among the most used pharmacological tools. Thanks to their availability countless numbers of thrombi and thrombemboli could be prevented and the morbidity related to cardiovascular events decreased. However, despite this success the application of anticoagulants is not without risk and serious sideeffects occur. Therefore the development of novel anticoagulants is anxiously awaited for. But the requirements for new anticoagulant drugs are stringent: they have to exhibit better efficacy, less sideeffects and a userfriendly mode of intake. New trends towards this goal (e.g. pentasaccharide, melagatran) might soon change the current standards of anticoagulation. This could affect the following indications: prophylaxis and therapy of deep vein thrombosis and pulmonary embolism, prophylaxis of stroke in atrial fibrillation, prophylaxis of thromboembolism in patients with mechanical heart valves, anticoagulation during pregnancy and antithrombotic therapy in acute coronary syndromes.

\section{Key Words}

anticoagulants - heparin - coumarin - factor Xa inhibitor - direct thrombin inhibitor - pentasaccharide melagatran

\section{Literatur beim Verfasser/Redaktion}

\author{
Anschrift für die Verfasser \\ PD Dr. Johannes Ruef \\ Medizinische Klinik III/Kardiologie, \\ Angiologie, Pulmologie \\ Gerinnungsambulanz und \\ Thrombophiliesprechstunde \\ Universität Heidelberg \\ Bergheimer Str. 58 \\ 69115 Heidelberg
}

\section{Grundlagen und mehr \\ Vitamin-Lexikon für Ärzte, Apotheker und Ernährungswissenschaftler}

Bässler K-H, Golly I, Loew D, Pietrzik $K, 2002$, 3. Auflage. 728 Seiten, 67 Abbildungen, gebunden, Urban $\mathcal{E} \mathrm{F}^{\prime} \mathrm{i}$ scher Verlag, München, Jena, ISBN 3437-21141-2

$\mathrm{M}$ ein letztes Werk und damit ist Schluss" war die Widmung in der neu erschienenen dritten Auflage des „Vitamin-Lexikons“, das ich von Karl-Heinz Bässler erhielt. Er hatte schrecklich Recht, denn wenige Monate später, im November des letzten Jahres, haben wir ihn zu Grabe getragen. Mit Karl-Heinz Bässler geht eine großartige Generation von physiologischen Chemikern zu Ende, die noch den Übergang sahen von den klassischen Arbeitsmethoden und Konzepten der physiologischen Chemie (als der biochemischen Grundlage lebender Prozesse) zu den so genannten molekularbiologischen Grundlagenforschungen im Bereich der biochemischen Medizin.

\section{Für Experten und für den}

\section{Anwender}

Das aktualisierte Vitamin-Lexikon ist eine Fundgrube an Daten und Erkenntnissen aus dieser faszinierenden Zeit der Entwicklung biochemischer Kenntnisse im Bereich der praktischen und theoretischen Medizin. Es bietet nicht nur eine systematische Darstellung der heutigen Erkenntnisse über die Vitamine von den Einzelbeschreibungen über Vitaminkombinationen bis zu den vitaminähnlichen Stoffen -, diese lexikalische Zusammenführung zeigt auch sehr praktische und zuverlässige Zugänge $\mathrm{zu}$ funktionellen $\mathrm{Zu}$ sammenhängen in den biochemischen Prozessen auf. Gerade Letztere sind für die klinische Medizin und damit für die Applikation von Vitaminen von großer Bedeutung. Sie sind in einer Weise aufbereitet, wie sie auch dem Nichtexperten genügend stabile Grundlagen vermitteln, mit dem Problem der Vitaminversorgung, der Beurteilung des Status und der therapeutischen Maßnahmen verantwortlich umgehen $\mathrm{zu}$ können.

Ein besonderes Gewicht legen die Autoren auf die Aspekte der Vitaminversorgung unter den besonderen Bedingungen chronischer oder altersbedingter Erkrankungen. Denn gerade mit zunehmendem Alter bedürfen in diesen Fällen sowohl die Referenzbereiche, auf die wir uns beziehen, als auch die Folgeerscheinungen von Defiziten einer neuen Betrachtung.

Für diesen Einstieg und den Gesamtüberblick liefert das vorliegende Werk eine exzeptionelle Grundlage und kann damit das Wissensbedürfnis sowohl der Experten, als auch der praktischen Anwender auf das Vorzüglichste abdecken. Die Darstellung ist komplett, präzise, außerordentlich detailliert und hervorragend belegt.

\section{Mehr als ein Lexikon}

Wir wünschen diesem Buch eine breite Anwendung, da die Daten in diesem Buch nicht nur dem speziellen Thema der Vitaminbiochemie gerecht werden, sondern darüber hinaus einen der seltenen gelungenen Ansätze untermauern, die Querverbindungen und die Gesamtzusammenhänge in den Lebensprozessen erkennbar werden zu lassen. Das Werk ist für die im Titel genannten Zielgruppen ganz sicher von hohem Gewinn - auch in der täglichen Arbeit. Es füllt ganz gewiss eine Lücke, die heute zunehmend durch die Spezialisierung und die auf molekularbiologische Prozesse gewichtete Darstellung des Lernmaterials auch für Studenten negative Auswirkungen hat. Die spezifische Benennung als „Vitaminlexikon“ sollte nicht verdecken, dass sich das Buch eben diesen Querbeziehungen und Gesamtzusammenhängen in hervorragender, stimulierender und auch gut geschriebener Form verschrieben hat.

Prof. Dr. Dr. Dr. h.c. A. Grünert, Ulm 